\begin{abstract}
UNIVERSIDADE DE BRASÍLIA
FACULDADE DE EDUCAÇÃO FÍSICA

CURSO DE LICENCIATURA EM EDUCAÇÃO FÍSICA UNIVERSIDADE ABERTA DO BRASIL - PÓLO BURITIS-MG
\end{abstract}

\title{
ANÁLISE DA ATIVIDADE FÍSICA E HÁBITOS ALIMENTARES NA EDUCAÇÃO FÍSICA INFANTIL: ESCOLA BALÃO MÁGICO EM URUANA DE MINAS-MG
}

ROMÁRIO PAULA ROCHA

BURITIS 
ROMÁRIO PAULA ROCHA

\section{ANÁLISE DA ATIVIDADE FÍSICA E HÁBITOS ALIMENTARES NA EDUCAÇÃO FÍSICA INFANTIL: ESCOLA BALÃO MÁGICO EM URUANA DE MINAS-MG}

Monografia apresentada como requisito final para aprovação na disciplina Trabalho de Conclusão de Curso II do Curso de Educação Física do Programa UAB da Universidade de Brasília - Polo Buritis.

ORIENTADOR: Prof. OSÉIAS GUIMARÃES DE CASTRO 


\section{DEDICÁTÓRIA}

Dedico esse trabalho primeiramente a Deus, por ter me concedido força e refúgio nos momentos difíceis, a minha família e amigos, que acreditaram no meu sucesso e sempre estiveram presentes me incentivando a seguir em frente. 


\section{AGRADECIMENTOS}

A Deus pelo dom da vida;

Aos meus pais pelo exemplo de vida, aos amigos e familiares pelo apoio e incentivo. Ao Samuel Silva Rocha pelo companheirismo.

Aos professores pelo conhecimento adquirido. 


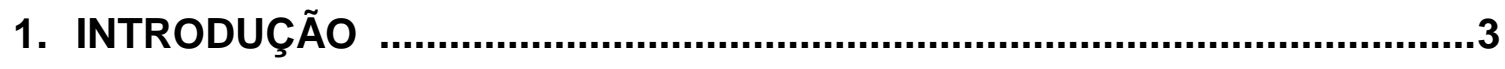

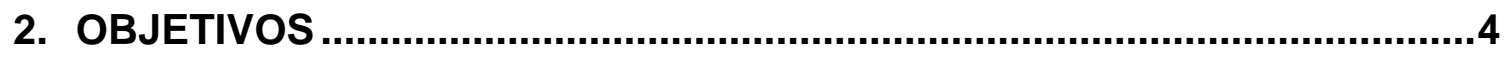

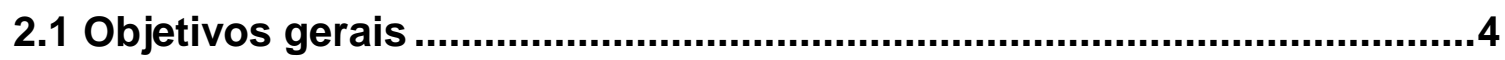

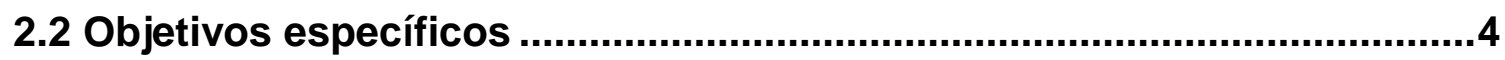

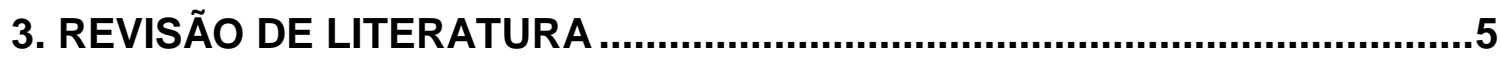

3.1 Conceitos de atividade física e hábitos alimentares ................................

3.2 Importância da família no comportamento alimentar e praticas de

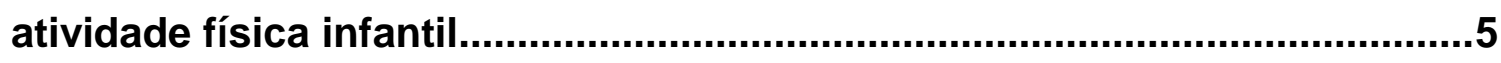

3.3 Hábitos saudáveis-Alimentação em grupo ...............................................6

3.4 Aspectos importantes para uma vida saudável familiar ..........................8

3.5 Influência da família no comportamento alimentar e pratica de atividade física infantil .................................................................................................11

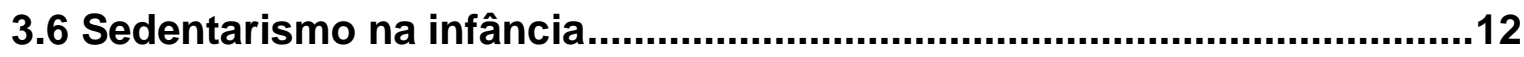

3.7 Fatores de risco

4. METODOLOGIA E DELINEAMENTO DE ESTUDO …...................................14

4.1 Apresentação e analise dos resultados....................................................14

5. CONCLUSÃO

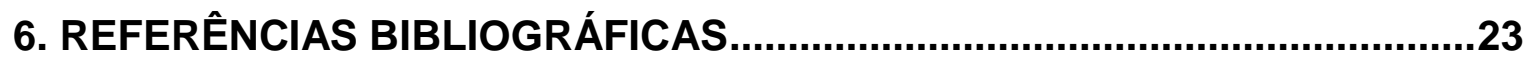

7. ANEXOS 
MONOGRAFIA: ANÁLISE DA ATIVIDADE FÍSICA E HÁBITOS ALIMENTARES NA EDUCAÇÃO FÍSICA INFANTIL: ESCOLA BALÃO MÁGICO EM URUANA DE MINAS-MG

AUTOR: ROMÁRIO PAULA ROCHA

\section{RESUMO}

Esse trabalho busca compreender por meio de uma revisão bibliográfica e de uma pesquisa de campo com os pais de alunos da escola Municipal Infantil Balão Mágico situada na Cidade de Uruana de Minas MG, de que forma eles atentam para o tema em questão, atividade física e hábitos alimentares na educação física infantil. Tendo como objetivos destacar a importância da família no processo de construção de hábitos saudáveis das crianças, a nível psicológico e motor, verificar ate que ponto a família pode intervir e modificar o contexto, diminuindo a sobrecarga exercida sobre a escola no controle da situação. Foi observado ainda que os pais conheçam e consomem alimentos nocivos, o que pode estar refletindo nos filhos em termos de má qualidade de vida, falta de atividade física relacionada ao sedentarismo e às facilidades da vida moderna também contribuem no processo desse grupo, refletir sobre os desafios encontrados e identificar os motivos porque eles se apresentam se faz necessário para buscar novas alternativas no combate ao sedentarismo. CONCLUSÃO: Após esse estudo verificamos que a praticidade para os pais se traduz em problemas de saúde para os filhos, muitos não incentivam a prática atividade física e ingestão de alimentos saudáveis como frutas e verduras regularmente, as ações de educação nutricional e alimentar devem ser permanentes. A mudança de hábitos, muitas vezes já enraizados, requer diálogo, reflexão e vigilância constantes.

Palavras chave: Níveis de atividade física, hábitos alimentares, infantil, sedentarismo. 


\section{1 - Introdução}

$\mathrm{Na}$ infância, a criança exerce pouco controle sobre sua disponibilidade de alimentos, sofrendo influência do hábito alimentar e de atividade física dos pais e familiares. Além disso, ela também está mais propensa a alterações de comportamento devido a sua inserção no ambiente escolar. $O$ ambiente familiar compartilhado e a influência dos pais nos padrões de estilo de vida dos filhos, incluindo a escolha dos alimentos, indicam o importante papel da família em relação ao futuro da criança. Uma revisão dos programas de prevenção da obesidade para crianças mostrou que as intervenções que produziram maiores efeitos incluíram a participação dos pais (SICHIERI, 2008).

Assim as crianças devem ser incentivadas desde pequenas a comerem alimentos saudáveis e praticar atividade física, o que não vem acontecendo de forma satisfatória, visto que os pais não estão tão presentes na seleção e oferta de alimentos e abordagem correta de atividade física às suas crianças. Os maus hábitos alimentares da família, a falta de tempo dos pais para a organização da alimentação da casa, as propagandas de alimentos nada saudáveis na TV, entre outros, contribui muito para o crescimento do número de crianças acima do peso saudável.

Portanto, a atividade física e hábitos alimentares representam parte importante para Educação Física na educação infantil ao ponto de chamar atenção para a temática do estudo: ANÁLISE DA ATIVIDADE FÍSICA E HÁBITOS ALIMENTARES NA EDUCAÇÃO FÍSICA INFANTIL: ESCOLA BALÃO MÁGICO EM URUANA DE MINAS-MG.

Segundo (Maciel 2004, p.26): Alimentação refere-se a um conjunto de substâncias que uma pessoa ou grupo costuma ingerir, implicando a produção e o consumo, técnicas e formas de aprovisionamento, de transformação e de ingestão de alimentos. Deste modo, alimentação vai além do biológico, relacionando com o social e a cultura.

Portanto, são temas que devem e precisam ser abordados com frequência no contexto escolar, pois, são fatores que garantem o sucesso ou deficiência no desenvolvimento da criança. Sabe-se que alimentação e atividade física representam parte importante na vida de qualquer pessoa, na infância eles devem 
ser priorizados, eles determinam a vida futura da criança, quando se trata de adeptos ou não a uma vida saudável.

Pensando assim Alves (2003 pag.27) nos faz analisar "que ser fisicamente ativo desde a infância possuem muitos benefícios não só na área física, mas também nas esferas sócio e emocional, e pode levar a um melhor controle das doenças crônicas da vida adulta". Além disso, pratica de atividade física melhora o desenvolvimento motor da criança, ajuda no seu crescimento e desenvolvimento estimulando assim sua participação futura em programas de atividade física.

\section{Objetivos}

\subsection{Objetivos Gerais}

Analisar a percepção e compressão dos pais de alunos da escola Infantil Balão Mágico com relação a atividade física e hábitos alimentares infantil.

\subsection{Objetivos Específicos}

$>$ Estudar os hábitos de atividade física e alimentares de 70 alunos do ensino infantil da Escola Municipal Balão Mágico, situada em Uruana de Minas - MG e relacionar de que forma os pais exercem influencia nas escolhas dos seus filhos neste contexto em questão.

Pesquisar qual a intensidade que crianças costumam realizar atividade física e a frequência com que consomem alimentos saudáveis direcionados e orientados pelos pais. 


\section{REVISÃO DE LITERATURA}

Abaixo destacaremos alguns pontos e subtemas importantes quando tratamos de níveis de atividade física e hábitos alimentares na educação física infantil, observando o contexto escolar.

Em 1985, Dietz e Gortmaker constataram a relação entre o tempo gasto assistindo à televisão e a prevalência de obesidade em crianças e adolescentes com idades entre 12 e 17 anos. $O$ estudo demonstra que o tempo em frente à TV influi diretamente no aumento da quantidade de gordura corporal em crianças e adolescentes.

Tucker (1986) estudou a relação entre o tempo de TV, aptidão física e obesidade em crianças e adolescentes. Sua pesquisa sugere que a prevalência de obesidade é positivamente proporcional ao tempo de TV e negativamente proporcional à aptidão física, em outras palavras, quanto maior o tempo de TV, maior será a prevalência de obesidade e menor sua aptidão física.

Dietz e Gortmaker (1985) citam ainda que a incidência da obesidade pode ser diminuída, e que este problema, na maioria dos casos, pode ser evitado com um aumento do tempo gasto em atividades fisicamente mais intensas.

\subsection{Conceitos de atividade Física e hábitos alimentares}

A atividade física trata-se a qualquer atividade ou exercício que tenha como consequência o gasto de energia e coloque em movimento uma grande quantidade de fenômenos a nível corporal, psíquico e emocional na pessoa que realiza. A atividade física pode ser realizada de maneira planejada e organizada, ou até mesmo, espontânea e involuntária, embora em ambos os casos os resultados apresentem-se de forma semelhantes.

\subsection{Importância da família na adoção de hábitos saudáveis dos filhos na infância}

O efeito oposto sobre a aceitação de alimentos pode ser obtido quando os responsáveis forçam as crianças a comer, por exemplo, legumes e verduras, a fim 
de ganhar recompensas. As crianças formam associações entre os alimentos e os contextos sociais em que a alimentação ocorre através da aprendizagem (BIRCH, 1999).

Geralmente, um controle excessivamente restrito e rígido por parte dos pais tende a prejudicar a capacidade da criança de se autorregular. Ao contrário, práticas de controles mais livres por parte dos pais promovem o desenvolvimento do amor próprio e do autocontrole nas crianças (BIRCH, 1999).

\subsection{Hábitos saudáveis - Alimentação em grupo}

As mudanças no estilo de vida familiares atuais levaram muitas crianças a passarem parte, ou a maioria de seus dias, em creches ou pré-escolas. Para muitas crianças, mais do que a metade dos nutrientes consumidos diariamente pode ser oferecida nestes estabelecimentos.

Os benefícios no consumo de frutas e hortaliças se expressam em diferentes formas na saúde humana. Estudos relatam sua importância para promoção da saúde e prevenção de doenças, proporcionando a redução dos teores energéticos totais, da densidade energética, e o fornecimento de vitaminas e minerais. [...] (TORAL; SLATER; SILVA, 2007)

Entretanto, não se tem claro o quanto o conhecimento interfere nos hábitos alimentares no contexto familiar, porém, acredita-se que conhecimento e competência sejam necessários para o estabelecimento de comportamentos desejados. Sabe-se que hábitos alimentares são difíceis de serem mudados, pois, além de envolverem mudanças em antigos padrões, são considerados componentes da história individual, da família e do grupo social (BARBOSA et al.,2013) .

Conforme podemos observar na figura1 a pirâmide alimentar contém os alimentos divididos em oito grupos, dos quais precisam estar em equilíbrio na alimentação de qualquer pessoa, independente da faixa etária, além disso, são divididos em quatro níveis, são eles: Energéticos extras, Construtores, Reguladores e Energéticos. 


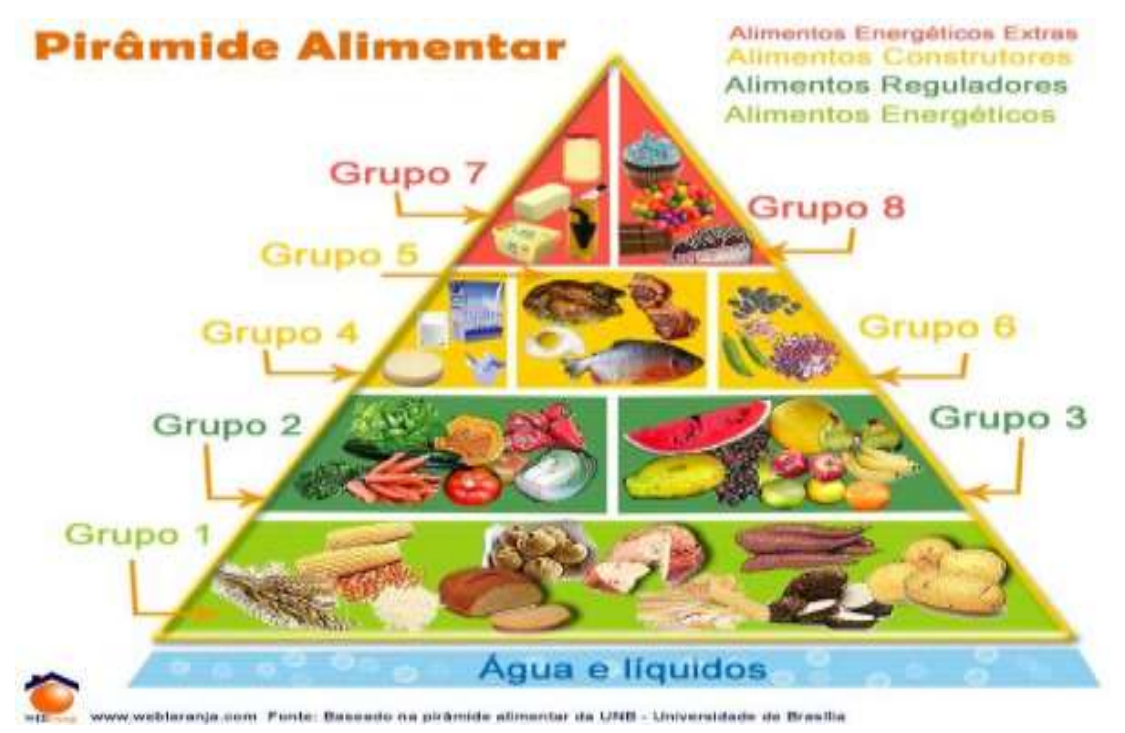

Figura 1: Piramide alimentar, nela contém os grupos alimentares, níveis e suas funções.

A respeito da água, os nutricionistas recomendam a ingestão diária de no mínimo 2 litros. Para eles, a água aparece na base da pirâmide configurando o alimento mais essencial para o ser humano. Com o intuito de enfatizar a teoria e de respaldar a prática, em 2012, a Coordenação Geral de Educação Alimentar e Nutricional do Ministério do Desenvolvimento Social e Combate à Fome realizou atividades para construir, de forma coletiva, o documento denominado Marco de Referência de Educação Alimentar e Nutricional para as Políticas Públicas (BRASIL, 2012), que adotou o termo "Educação Alimentar e Nutricional" (EAN), definindo-o como:

[...] campo de conhecimento e de prática contínua e permanente, transdisciplinar e multiprofissional que visa promover a prática autônoma e voluntária de hábitos alimentares saudáveis. A prática da EAN deve fazer uso de abordagens e recursos educacionais problematizadores e ativos que favoreçam o diálogo junto a indivíduos e grupos populacionais [...] (BRASIL, 2012, p. 23).

A escola é um local privilegiado de intervenção, onde pequenas mudanças, ao nível da alimentação e atividade física, podem contribuir para travar a escalada da obesidade e conduzir a estilos de vida mais saudáveis, no entanto, os pais devem contribuir para adoção de práticas saudáveis para que reflita sobre as escolhas dos seus filhos. Também se baseando na constatação de não correspondência à expectativa, fato que compromete a ideia de reforçar a 
importância da EAN no contexto da Promoção da Saúde - o marco amplia a responsabilidade das ações educativas sobre alimentação e nutrição para outros profissionais da saúde, buscando aumentar a ocorrência e a visibilidade das experiências em diferentes campos de atuação (BRASIL, 2012).

Entende-se que isso seja uma consequência histórica da falta de uma definição clara e objetiva da EAN e da insuficiência de respaldo teórico para o embasamento de suas práticas, tendo, pois, constituído inquietações que regeram a elaboração do marco (BRASIL, 2012).

\subsection{Aspectos importantes para uma vida saudável familiar}

Vários fatores influenciam o comportamento alimentar, entre eles fatores externos (unidade familiar e suas características, atitudes de pais e amigos, valores sociais e culturais, mídia, alimentos rápidos, conhecimentos de nutrição e manias alimentares) e fatores internos (necessidades e características psicológicas, imagem corporal, valores e experiências pessoais, autoestima, preferências alimentares, saúde e desenvolvimento psicológico). Vale ressaltar também que a perspectiva da alimentação saudável, presente nos demais cenários, é entendida, no marco, como uma estratégia de enfrentamento dos novos desafios no campo da saúde, prevista a todos os profissionais da área, inclusive aos nutricionistas da saúde, à medida que a prática de hábitos alimentares saudáveis é inerente a qualquer contexto que vise promover saúde (BRASIL, 2012).

A dificuldade em estabelecer um bom controle de saciedade é um fator de risco para desenvolver obesidade, tanto na infância quanto na vida adulta. Boog (2013) refere que as ações educativas de alimentação e nutrição devem ser procedidas de um diagnóstico educativo, diferente do diagnóstico de saúde como os apresentados na maioria dos artigos estudados.

Quando as crianças são obrigadas a comer tudo o que é servido, elas podem perder o ponto da saciedade, por isso deve-se ter muita cautela quando vai oferecer um alimento para criança. Os pais exercem uma forte influência sobre a ingestão de alimentos pelas crianças, entretanto, quanto mais os pais insistem no consumo de certos alimentos, menor a probabilidade de que elas os consumam. Da mesma forma, a restrição por parte dos pais pode ter efeito deletério. Na primeira infância, 
recomenda-se que os pais forneçam às crianças refeições e lanches saudáveis, balanceados, com nutrientes adequados e que permitam às crianças escolher a qualidade e a quantidade que elas desejam comer desses alimentos saudáveis. Diminuir o consumo de alimentos e preparações hipercalóricas já é suficiente para a redução do peso.

O processo de urbanização acompanhou-se de importantes mudanças sociais, tais como: rearranjos familiares, a inserção da mulher na sociedade, incrementos tecnológicos, entre outras. Também o padrão demográfico alterou-se, sendo atualmente uma população essencialmente urbana (DUARTE; BARRETO, 2012). A transição epidemiológica ocorre desde os últimos anos, caracterizada pela gradual e progressiva diminuição das doenças infecciosas e parasitárias e pela ascensão das doenças crônico-degenerativas.

Nesse sentido, a comida adquiriu uma identidade, traduz e representa a sociedade em questão. Dessa forma, é possível caracterizar as sociedades conforme seus hábitos e escolhas alimentares (ROMANELLI, 2006; SANTANA, 2012). A universalização da escola não pode ser tratada sem considerar o papel que a instituição escolar desempenha na sociedade, desse modo, a escola pode ser pensada de forma organizada e sistemática na transmissão de seus conhecimentos e valores, o que não seria possível transmitir em nível familiar ou individual.

Dessa forma, temos os hábitos alimentares e a falta de atividade física como os principais fatores para o aumento do sobrepeso e obesidade em todas as idades, ressaltando que estes já alcançaram proporções epidêmicas nos países desenvolvidos (GUEDES; GUEDES, 1998; DAMIANI; CARVALHO; OLIVEIRA, 2000).

Falar em obesidade num país onde se estima que 46 milhões de pessoas passem fome e $6 \%$ é desnutrido pode parecer ironia, mas a situação no Brasil é: $40 \%$ da população é obesa e desse percentual, $15 \%$ são crianças e se essa situação não for contornada, o número de obesos continuará aumentando, podendo atingir cerca de 70 milhões de pessoas. Os termos publicidade e propaganda aplicada à área de alimentação, segundo Beiler (2012, p. 483)

"[...] possuem o mesmo significado, abrangendo um conjunto de técnicas e atividade de informação persuasão, com fins ideológicos ou comerciais, 
utilizadas com objetivo de divulgar conhecimento e/ou visando exercer influência sobre o público, por meio de ações que visem promover e/ou induzirem a prescrição, a aquisição, a utilização e o consumo de alimentos".

Essa população tem se tornado cada vez mais sedentária, uma vez que cada dia as cidades se tornam mais violentas e as crianças trocam as brincadeiras saudáveis por vídeo games, computadores e televisões. Muitas são as causas, Oliveira (2003) diz que as causas da obesidade na infância são de caráter multifatorial, envolvendo fatores biológicos, psicológicos e ambientais. Dentre esses fatores, o ambiental, que envolve a inatividade física e hábitos alimentares familiares, tem se mostrado um fator determinante.

Além disso, os governos através das regulamentações devem estar dispostos a se aproximar da realidade e, reforçar estratégias para os direitos de alimentação adequada, para que o indivíduo possa gozar da sua liberdade de escolha alimentar de forma saudável (VASCONCELLOS, 2014).

O estilo de vida e o exemplo de pais e educadores são o que podem manipular para tratar e prevenir a obesidade infantil. Mudar o hábito alimentar, incentivar as crianças e adolescentes a optarem por algum tipo de atividade física ao invés de criar hábitos sedentários desde cedo, leva o profissional de educação física a ter um grande papel na prevenção desse mal que nos cercam.

O que complementa a percepção de Beiler e Cols ao afirmar que já dispomos de evidências suficientes para dizer que a mídia televisiva influencia as preferências alimentares da sociedade. Com isso, e outros aspectos determinantes, as propagandas vêm contribuindo para um ambiente promotor de obesidade, também chamado de "ambiente obeso gênico", o qual apresenta acesso amplo e facilitado a alimentos de alta densidade energética, pobres em micronutrientes, a exemplo dos modernos alimentos processados os quais apresentam altos teores de gorduras, açúcar e sódio, geralmente consumidos fora do ambiente familiar, mas também presentes no domicílio uma vez que emprega tempo e facilidade no preparo. (VASCONCELLOS, 2014; TORAL, 2007; BEILER, 2012).

Complementando citações anteriores relativas à época primitiva, em que o homem buscava alimento pra suprir demandas biológicas, com a evolução da 
espécie, nosso sistema cerebral desenvolveu mecanismos dedicados a escolha dos alimentos, que inclui além da necessidade, também procura atender necessidades sensoriais e psicológicas (PRESCOTT, 2012; LANDEIRO, 2011).

\subsection{Influência da família no comportamento alimentar e prática de atividade física infantil}

Considerando a influência do meio ambiente sobre o desenvolvimento do excesso de peso em nosso meio e a influência do estilo de vida ambiente familiar. $O$ risco de uma criança se tornar obesa quando nenhum dos pais é obeso, é de $9 \%$ quando um deles é obeso o risco se eleva para $50 \%$ e quando os dois são obesos a porcentagem chega aos $80 \%$ (MOREIRA et al., 2014).

O sistema de recompensa tem relevância, visto que pode determinar a intenção alimentar e, muitas vezes, se sobrepor a saciedade, interferindo inclusive na regulação fisiológica da fome. As escolhas por preferência garantem um bemestar e recompensa afetiva e psicológica (ROLLS, 2011; PRESCOTT, 2012).

Assim, o exposto indica a forte relação existente os fatores neurais, fisiológicos, endócrinos e comportamentais relacionados ao sistema de saciedade, fome e, por conseguinte, recompensa, no que tange à alimentação (PRESCOTT, 2012). Dessa maneira o indivíduo deve mudar seus hábitos de vida, como: ser mais ativo, substituindo rotinas tecnológicas, com pouca perda calórica por estilo de vida com mais atividades como, por exemplo, trocar o tempo de televisão diária por uma caminhada na rua; reduzir a quantidade de gordura ingerida reforçando o que acima foi exposto.

A obesidade, os excessos alimentares e o sedentarismo são sintomas gritantes de sofrimento emocional, é preciso verificar com cuidado a psicodinâmica familiar, o vínculo mãe-filho e o meio ambiente muitas vezes doentio e perverso onde vivem tais crianças (ANDRADE; MORAES; LOPES, 2012).

Nesse sentido, também a ausência dos pais no ambiente familiar, contribuem para depositar a responsabilidades destes, no mundo globalizado, no estresse das grandes cidades ou por simples praticidade. É oferecida a criança alimentos industrializados, além de não controlar as preferencias infantis por alimentos ricos em açúcar (MEDEIROS et al., 2012). Vale lembrar que nossas crianças são mais 
susceptíveis a esse processo, pois são educados dentro dessa cultura de excesso de alimentos e pouca atividade física.

Um fator importante para a instalação do sobrepeso/obesidade infantil é a insegurança e a falta de espaços apropriados e adequados para que as crianças possam brincar correr, pular e queimar as calorias e energias acumuladas na sua rotina diária. Hoje, as crianças, basicamente, resumem suas brincadeiras a computadores/jogos, videogame e televisão/DVD. Um dos fatores que contribuem é o fato de que o avanço tecnológico, as cidades tornaram-se mais violentas, fazendo com que as crianças trocassem brincadeiras saudáveis por TVs e jogos eletrônicos. (OLIVEIRA, 2013).

Com isso, passam a maior parte do dia sentado ou deitado, e na maior parte do tempo comendo alguma coisa.

Outros pontos que merecem atenção são o medo e a violência urbana, fatores as quais as crianças de nossa sociedade tem sido constantemente expostas, e que geram uma ansiedade que normalmente é compensado-descontada no ato de comer. Em função desse medo, as crianças estão cada vez mais isoladas e gordas, uma vez que a comida se torna, em alguns casos, uma forma de proteção e a principal companheira, além de trazer satisfação imediata aos seus anseios.

\subsection{Sedentarismo na Infância}

O controle deste problema obesidade deve ser iniciado já na infância e, como a causa mais comum de sobrepeso e obesidade se deve ao aumento da ingesta calórica, associada à falta de atividade física regular. De acordo com a Organização Mundial da Saúde (OMS), a alimentação e a nutrição saudável constituem um dos determinantes para a melhoria da saúde da população e em especial das doenças crônicas não transmissíveis (JAIME et al., 2015).

A literatura aponta que, a obesidade é decorrente de patologias endócrinas ou genéticas em apenas $1 \%$ dos casos, enquanto que os $99 \%$ restantes são considerados de causa exógena, ou seja, resultantes do consumo excessivo de alimentos, a inserção do contexto familiar contribui para estes dados (BERTOLETTI; SANTOS, 2012).

\subsection{Fatores de Risco}


Segundo Halpernapud Colloca e Duarte (2015) a obesidade, pode ser identificada conforme à forma que se apresenta no corpo, dependendo de como a gordura é distribuída, pode ser classificada como obesidade Androide e Ginoide. Chamada de obesidade androide, conhecida também como obesidade em forma de maçã ou obesidade visceral, quando a gordura está concentrada no tronco, abdômen e tórax, os membros superiores e inferiores tendem à ser menos volumosos, com maior predominância nos homens.

Esta pode estar associada à outras doenças (intolerância à glicose, hiperlipidemia, complicações cardiovasculares e acidente vascular cerebral). Já a obesidade Ginoide, predominante em mulheres e associada à forma de pera, concentra maior quantidade de gordura nas nádegas, coxas, abdômen. No entanto diferente da obesidade androide que concentra a gordura entre as vísceras, esta apresenta maior quantidade de gordura na parede (HALPEN apud COLLOCA; DUARTE, 2015).

A obesidade infantil é uma patologia mais complexa do que parece, seus fatores de risco podem ser classificados em endógenos (internos ao organismo) e exógenos (externos ao organismo).

Fatores endógenos: podem estar relacionados á alterações hormonais e disfunção na tireoide, a carga genética também tem relevância na evolução da obesidade em uma criança.

Os fatores exógenos: constituem o principal motivo pelo qual ocorre o surgimento da obesidade, são fatores relacionados ao estilo de vida, portanto, são modificáveis.

Fatores endógenos (genéticos, neuropsicológicos, endócrinos, metabólicos) representam $5 \%$, dos casos de sobrepeso e obesidade. Já os fatores exógenos (externos), (origem comportamental, dietética e/ou ambiental) representam cerca de $95 \%$ dos casos. Dentre os fatores exógenos que contribuem para estados de sobrepeso e obesidade infantil, as pesquisas não apontam, ou pelo menos não existe consenso, que indique um fator preponderante, reforçando a ideia da multicausalidade (DÂMASO et al.,apud COLLOCA; DUARTE, 2015) 


\section{Metodologia e Delineamento do Estudo}

Trata-se de uma pesquisa de abordagem qualitativa do tipo exploratória realizado com os pais dos alunos com idade média entre 4 e 5 anos da escola Municipal Balão Mágico da cidade de Uruana de Minas- MG, 70 questionários foram respondidos.

Do total dos entrevistados que aceitaram responder o questionário 50 eram mães, 10 eram avós das crianças e os outros 10 eram pais, parte residem na zona urbana já os outros moram na área rural do município.

Os dados foram coletados por meio de um questionário estruturado fechado com sete questões estruturadas pelo entrevistador sobre o tema em conformidade com a realidade local. De acordo com Santos (2009) a coleta dos dados sendo realizada dessa forma, o entrevistador tem maior controle dos resultados.

A pesquisa primou pelos aspectos éticos, que disserta sobre a realização de pesquisas com seres humanos. Desta forma, foi solicitada a assinatura do Termo de Consentimento Livre e Esclarecido (TCLE), onde foi assegurado o anonimato dos participantes.

Os entrevistados foram orientados e informados previamente por meio de comunicado expedido com o auxílio da escola via telefone e recadinho enviados pelos alunos.

A pesquisa durou nove dias, ocorrendo entre os dias 01 a 09 de Agosto de 2017 e contou com a contribuição e consentimento da direção da escola que cedeu o espaço para aplicar o questionário.

\subsection{Apresentação e Análise dos Resultados}

Os dados foram tabulados e computados após analisados em ação conjunta com o professor regente de educação física e nutricionista da Escola Municipal Balão Mágico de Uruana de Minas com crianças entre 4 e 5 anos e seus respectivos pais.

Apresentaremos nessa seção os dados encontrados na pesquisa qualitativa, descrevendo cada um dos pontos encontrados através do questionário aplicado e citado na seção 4 e que se encontra disponível no Anexo I ao final deste trabalho. Sendo os entrevistados compostos por um percentual de $42 \%$ do sexo masculino e $58 \%$ do sexo feminino. 
É importante destacar a atuação dos pais nesse processo de busca por respostas, com eles o projeto ganhou mais clareza, mesmo realizado inteiramente no contexto escolar, o projeto considerou que as crianças também praticam atividade física fora da escola, do mesmo modo que se alimentam isso foi importante em nível de comparação com os resultados comparados enquanto período escolar.

Após aplicação de questionário do tipo fechado, utilizamos como recurso o programa Microsoft Excel do Windows 8 para análise dos resultados e encontramos os seguintes resultados.

Analisando a questão de número um do questionário foi possível compreender que $05 \%$ do total de 70 alunos sofrem de algum tipo de doença ocasionada pelo mal habito de vida, em sua maioria, a obesidade, relatam os pais, do total $02 \%$ são meninas e $03 \%$ meninos (dados acompanhados e obtidos com o auxílio da nutricionista da escola) conforme podemos observar no gráfico 1.

Com relação ao estilo de vida, as pessoas vêm se tornando cada vez mais sedentários, devido, principalmente, as comodidades oferecidas pelo mundo moderno, tendo uma grande influência nas possíveis causas de doenças, como a obesidade, sobrepeso, dentre outras (GARCÍA et al, 2013).

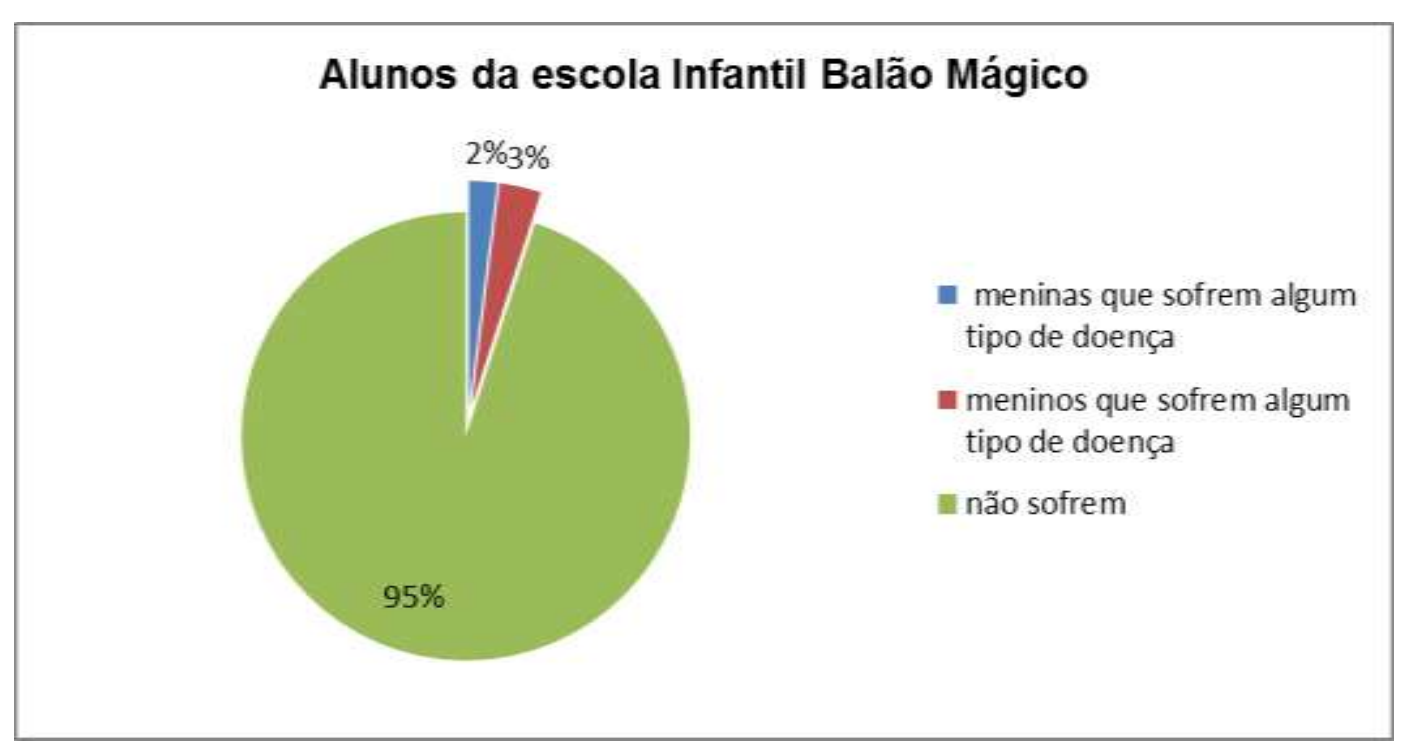

Gráfico 1: Alunos que sofrem algum tipo de doença relacionados a má qualidade alimentar e falta de atividade física adequados.

De acordo com a Organização Mundial de Saúde (OMS) (2012) e após observar o gráfico de numero um, o sobrepeso e a obesidade são grandes fatores 
de risco para uma série de doenças crônicas, como diabetes, doenças cardiovasculares e câncer. Uma vez considerado um problema apenas dos países de renda elevada, o número de pessoas com excesso de peso ou obesidade está aumentando drasticamente em países de baixa e média renda, especialmente em áreas urbanas.

Os resultados obtidos com as questões 2, 3, 4 e 5 fazem atentar para relação existente entre os níveis habituais de atividade física e alimentação equilibrada são fatores intervenientes na manutenção de uma vida saudável, onde controle alimentar e a prática de atividade física são comprovadamente formas de atuar contra doenças crônicas não transmissíveis, porém, apesar de a população ter consciência dos benefícios desses hábitos saudáveis, parece haver, em todos os níveis populacionais, certa resistência na efetiva adesão a estas práticas (FIN; BARETTA; NODARI JÚNIOR, 2013).

$\mathrm{Na}$ questão dois do questionário as crianças passam em média 5 horas por dia na escola, tempo estimado tanto para meninos quanto para meninas. Quanto ao grau de pouco satisfeito (PS), satisfeito (S) e muito satisfeito (MS) presente nas alternativas da questão 3 do questionário, na letra $(A)$ que é relativo as aulas de educação que são oferecidas na escola, $5 \%$ dos pais estão (PS), 35\% (S) e $60 \%(\mathrm{MS})$, a letra (B) referente ao nível de atividade física oferecida pelo professor e a escola, $5 \%$ responderam (PS), $45 \%$ (S) e 50\% (MS), sobre as letras C e D da mesma questão referente a alimentação que é oferecida na escola e Serviços de apoio de nutrição/alimentação, 3\% responderam (PS), 37\% (S) e 60\% (MS) (Observe os dados na tabela 1).

\begin{tabular}{|l|l|l|}
\hline $\begin{array}{l}\text { Nível de satisfação das } \\
\text { aulas de Educação } \\
\text { Física oferecidas na de atividade física } \\
\text { escola. }\end{array}$ & $\begin{array}{l}\text { Alimentação oferecida } \\
\text { oferecida pelo professor } \\
\text { e escola. }\end{array}$ & $\begin{array}{l}\text { la escola e serviços de } \\
\text { apoio de nutrição/ } \\
\text { alimentação. }\end{array}$ \\
\hline Pouco Satisfeito: $5 \%$ & Pouco Satisfeito: $5 \%$ & Pouco Satisfeito: $3 \%$ \\
\hline Satisfeito: $35 \%$ & Satisfeito: $45 \%$ & Satisfeito: $37 \%$ \\
\hline Muito satisfeito: $60 \%$ & Muito satisfeito: $50 \%$ & Muito satisfeito: $60 \%$ \\
\hline
\end{tabular}

Tabela 1: Níveis de satisfação dos pais referente às aulas de educação física oferecida pela escola e alimentação. 
Se para o indivíduo adulto conviver com as dificuldades vivenciadas através da obesidade, para a criança portadora da doença aumenta ainda mais as dificuldades. Quanto a isso (PIMENTA; ROCHA; MARCONE, 2015) destacaram em seu estudo que tantos desafios numa fase inicial das vidas destes indivíduos são enfrentados como um meio para o alcance da aceitação social, tendo em vista a importância deste fator como promotor da qualidade de vida do ser humano.

Diante disso, podemos considerar que os avaliados estão sendo muito bem assistidos por profissionais habilitados com programas alimentares e de atividade físico satisfatórios para atender as crianças da escola Infantil Balão Mágico.

Em contrapartida, devemos considerar que os hábitos alimentares de determinada população precisam ser alterados, no entanto trata-se de um grande desafio para as políticas públicas e para a educação em nutrição, dado que esses envolvem relações entre pessoas e comportamentos humanos (BOOG, 2013).

Quando perguntado aos pais sobre de que forma a criança se desloca ate a escola, referente a questão 4 do questionário, $40 \%$ responderam á pé, $50 \%$ de bicicleta e $10 \%$ responderam que as crianças chegam até a escola de carro, (observe a tabela 2 ).

Estes dados são resultados da realidade local da cidade, devido ser muito pequeno e a distancia das residências serem próximas, muitos dos pais optam por levarem seus filhos a pé ou de bicicleta, poucos levam seus filhos de carro ou chegam de ônibus escolar, é assim que incentivamo-los a adotar e seguir uma vida mais saudável, tirando-os do cenário do sedentarismo, destacou a maioria dos pais entrevistados.

Os resultados aqui encontrados em relação ao nível de atividade física dos escolares difere dos estudos de Dresch et al (2013). Corroborando com nossos achados o estudo de Mello et al (2014), ao avaliar o nível de atividade física de escolares de instituições públicas utilizando os mesmos procedimentos, analisou que $68,0 \%$ eram inativos e $23,7 \%$ irregularmente ativo, apresentando como ativos só $7,6 \%$ dos escolares, resultados estes reforçam a importância de ações para o aumento do nível de atividade física de crianças e adolescentes, onde estes comportamentos parece exercer efeito protetor contra fatores de risco para diferentes problemas de saúde nesta faixa etária. 
Os pais são e devem propor alternativas para que seus filhos sejam ativos, a atividade física além de promover melhores condições para uma vida futura e no presente saudava melhoram a percepção das crianças, em se tornarem também adultos ativos no sentido de atividade física e bons hábitos alimentares.

$\mathrm{Na}$ questão cinco do questionário, perguntamos aos pais quanto tempo a criança, independente do sexo, se dedica em média (minutos ou horas), a ver TV, jogar no celular ou vídeo game ao longo da semana.

Do total, $40 \%$ responderam que as crianças passam em média 50 min ao longo da semana brincando em algum desses aparelhos tecnológicos, já os outros $60 \%$ dos pais relatam que seus filhos passam em média 2 horas ao longo da semana dedicados aos jogos ou programas oferecidos pelos aparelhos digitais, (observe a tabela 2).

Vale ressaltar que esse tipo de comportamento deve ser coibido pelos pais, pois, as crianças passam muito tempo a frente dessas máquinas ao invés de estarem se dedicando a alguma atividade que estimule o gosto por atividades saudáveis, ou seja, precisam brincar mais se movimentando e gastando energia e não ganhando excesso de peso por ficarem paradas manipulando tais objetos. Com relação as atividades, a mesma favorece o processo de aprendizagem da criança, facilitando o desenvolvimento da linguagem, do pensamento, da socialização, da iniciativa e da autoestima (MOREIRA, 2013).

De acordo com Pandolfo et al (2014), o crescente aumento dos níveis de obesidade em crianças e adolescentes vem se configurando em um problema de saúde pública, tendo em vista que o excesso de gordura é um indicador para o desenvolvimento e agravamento de alterações metabólicas. Entretanto, mesmo com a crescente valorização da educação nutricional nos últimos anos, tem-se o consenso da dificuldade de desenvolver intervenções em decorrência da baixa existência de referenciais teóricos, metodológicos e operacionais (SANTOS, 2012).

\begin{tabular}{|l|l|}
\hline $\begin{array}{l}\text { De que forma a criança se desloca } \\
\text { até a escola }\end{array}$ & $\begin{array}{l}\text { Quanto tempo a criança se dedica } \\
\text { (min/horas) a ver TV, jogar em celular } \\
\text { ou vídeo game ao longo da semana. }\end{array}$ \\
\hline A pé: $40 \%$ & Min/ semana: $40 \%$ \\
\hline
\end{tabular}




\begin{tabular}{|l|l|}
\hline Bicicleta: $50 \%$ & Horas/ semana: $60 \%$ \\
\hline Carro/Ônibus: $10 \%$ & \\
\hline
\end{tabular}

Tabela 2: formas de deslocamento até a escola e quantidade de tempo ao longo da semana que a criança se dedica a ver TV, jogar em celular e vídeo game.

Vale ressaltar também que a perspectiva da alimentação saudável, presente nos demais cenários, é entendida, no marco, como uma estratégia de enfrentamento dos novos desafios no campo da saúde, prevista a todos os profissionais da área, inclusive aos nutricionistas da saúde, à medida que a prática de hábitos alimentares saudáveis é inerente a qualquer contexto que vise promover saúde (BR ASIL, 2012).

$\mathrm{Na}$ questão seis perguntamos aos entrevistados quantas vezes por mês, normalmente o seu filho/a come fora de casa. (não contar com as refeições da escola). Segundo Rodrigues et al (2015) a maior preocupação do crescimento da população infantil obesa é o aumento das morbidades e problemas de saúde na vida adulta. Verificar o perfil genético familiar e identificar risco para o desenvolvimento da obesidade infantil faz-se necessário para que se possa traçar um plano de cuidados que vise à diminuição do risco para o aparecimento da doença nas crianças.

Em resposta, $10 \%$ dos pais relataram que seus filhos comem pelo menos 15 vezes ao longo do mês em casa de familiares, os outros $90 \%$ comem pelo menos 25 vezes ao longo do mês em algum lugar que ofereça comidas de fast-food, pizzaria ou lanchonete, além de restaurante, (Observe o gráfico 2).

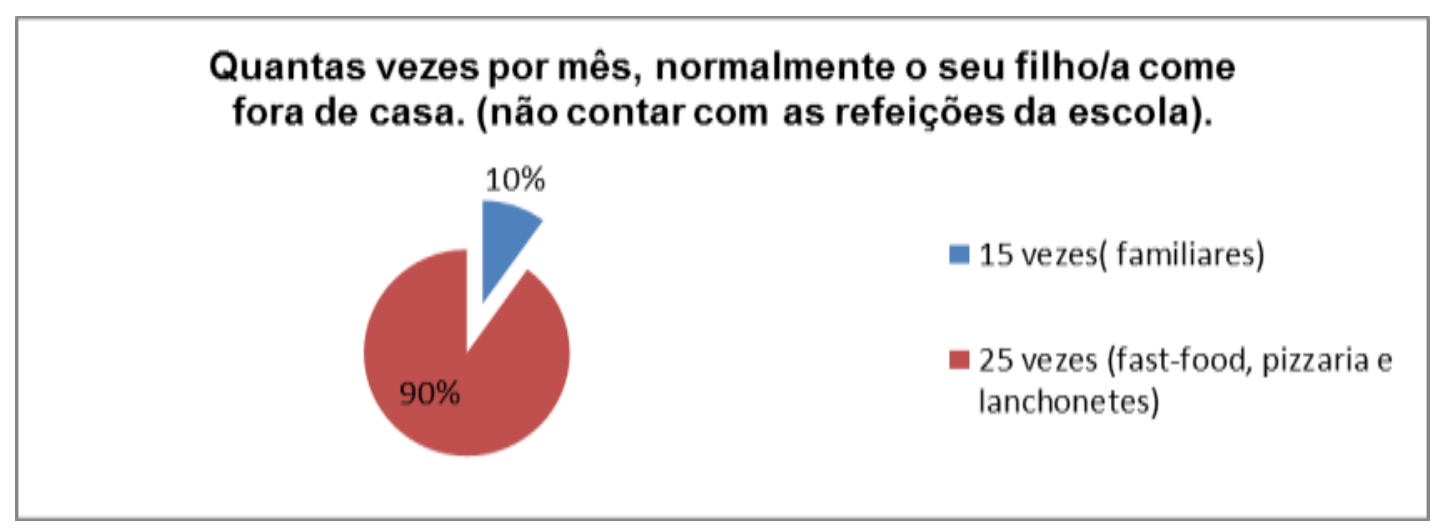

Gráfico 2: Quantidade mensal que as crianças comem fora de casa

Mais uma vez observamos que os pais contribuem significativamente na alimentação dos filhos, vem deles a responsabilidade de adotar medidas para 
prevenir a ingesta de alimentos calóricos pobre em nutrientes, nos dados obtidos com a questão seis do questionário entendemos que os pais pouco importam com a vida nutritiva da criança, mesmo sabendo que nessa fase devemos ofertar o maior numero de vezes alimentos mais saudáveis e extinguir produtos industrializados.

Gomes, Dezan e Barbieri (2014) apresentaram em seu artigo uma estimativa da Organização Mundial da Saúde, que no mundo, aproximadamente $10 \%$ dos indivíduos entre 4 e 5 anos estejam com excesso de gordura corporal, sendo que $2 \%$ a $3 \%$ são obesos, correspondendo a 155 milhões de crianças com excesso de peso e de 30 a 45 milhões de obesas.

Lobstein, Baur e Uauy apud Gomes, Dezan e Barbieri (2014), constataram dados preocupantes, comparando a prevalência de crianças acima do peso em alguns países, na China uma em cada treze crianças está acima do peso, no Brasil uma em cada sete, e na Itália uma em cada três.

A Atividade Física na infância isoladamente não resolve o problema apenas contribui para tornar um adulto mais motivado e disciplinado fisicamente. A atividade física e incorporação de hábitos alimentares saudáveis se complementam e são fundamentais à redução da obesidade infantil e controle da hipertensão e diabetes, bastante comuns em crianças com percentual de gordura corporal elevada. É importante lembrarmos a participação do nutricionista nesse processo, sendo o mesmo respaldado pela lei $n$ ㅇ 8324/91.

Uma boa alimentação é aquela que prevê a ingestão de alimentos variados e em quantidade adequada, garantido que o corpo receba todos os nutrientes necessários para a realização das atividades diárias. Para manter o corpo em funcionamento é essencial que haja a ingestão de alimentos importantes que contêm nutrientes como carboidratos (amidos e açúcares), gorduras (lipídios), proteínas, água, sais minerais e fibras. O consumo equilibrado dos nutrientes possibilitará melhor saúde e qualidade de vida.

Em seu estudo Penteado Silva e Bittar (2012) concordam afirmando que vivemos uma situação conhecida como transição nutricional, que é caracterizada pela inversão da distribuição dos problemas nutricionais da população, sendo geralmente uma passagem da desnutrição para a obesidade.

Junto a esta questão destacamos os meios de comunicação, a publicidade de alimentos é um fator que tem colaborado para o consumo indiscriminado de 
alimentos inadequados, em razão do excesso de açúcares, sal e gorduras que favorecem o desenvolvimento das doenças crônicas como diabetes, hipertensão e obesidade. Segundo Henriques et al., (2012), a veiculação de propagandas de alimentos inadequados para crianças tem sido cada vez mais cedo, sendo as crianças expostas a elas seja pela ausência dos pai em decorrência da jornada de trabalho ou pela falta de opções saudáveis nas escolas.

$\mathrm{Na}$ ultima questão, a de número sete do questionário, perguntamos se durante o último ano teve dificuldade para conseguir que a criança comesse o que desejava para ela saudável (considere frutas, verduras e legumes) no período em que não esta na escola.

Corroborando com nossa fala Giordani; Almeida; Pacheco, (2013) afirmam que a qualidade do cuidado, nos aspectos físico e afetivo-social, decorre de condições estáveis de vida, tanto socioeconômicas quanto psicossociais. De acordo com Moraes e Dias (2012), nos dias atuais espera-se que a família seja a principal responsável pelo desenvolvimento da criança, dessa forma assegurando-a a transmissão de afetos, acolhimento, bem-estar, promovendo proteção e segurança aos seus integrantes.

Relacionando o que foi discutido ate aqui o resultado já era esperado, 90\% dos pais disseram ter havido muita dificuldade e propor alimentos saudáveis aos seus filhos e 10\% apenas responderam que não tiveram dificuldade, essa minoria destacou que são os pais que devem primeiramente ensinar aos filhos o que é saudável, não apenas ensinar, mas dar exemplos de atitudes saudáveis, (Observe o gráfico 3).

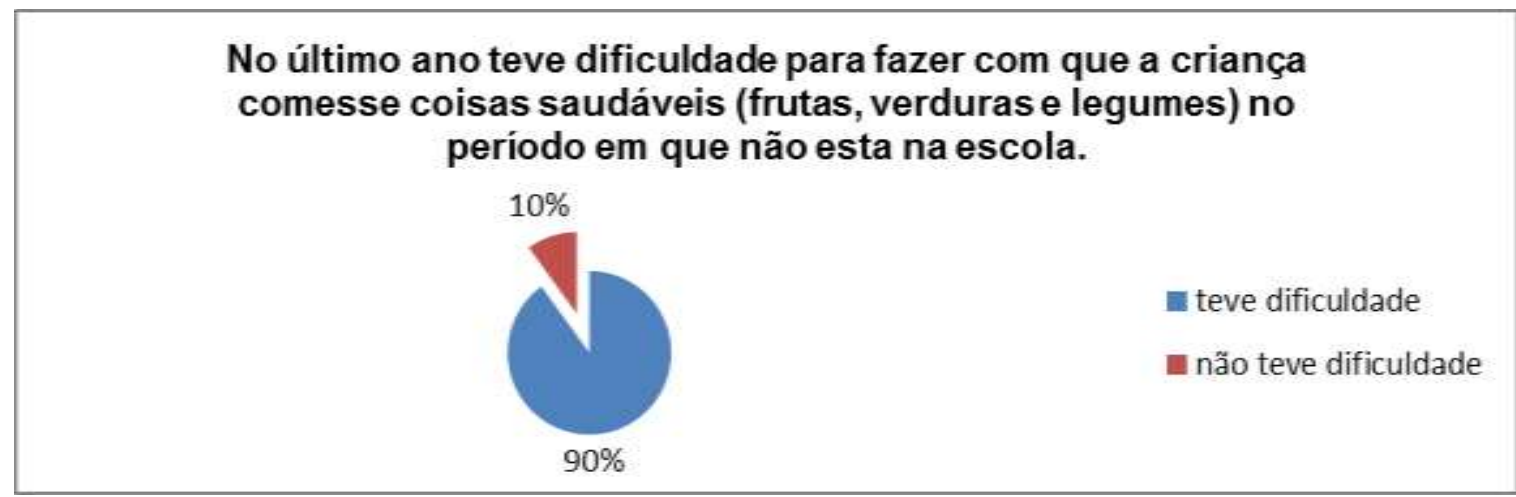

Gráfico 3: Reativo a dificuldade dos pais em introduzir na alimentação das crianças frutas, verduras e legumes fora do contexto escolar. 
Todos nos sabemos que as crianças pertencem ao grupo com pior perfil de dieta, com as menores frequências de consumo de feijão, saladas e verduras em geral, apontando para um prognóstico de aumento do excesso de peso e doenças crônicas. Esse comportamento alimentar retrata um perfil epidemiológico preocupante, estima-se que cerca de $70 \%$ dos brasileiros estarão com excesso de peso, em vinte anos, no Brasil (BRASIL, 2012), sendo urgente a criação de ações para o controle e prevenção do ganho de peso. E os hábitos alimentares inadequados dos pais têm sido alguns dos principais responsáveis pelo aumento da prevalência da obesidade infantil (SENA; PRADO, 2012).

É interessante destacar também que os entrevistados lutam para direcionar as crianças, no entanto, elas têm adquirido desde cedo hábitos alimentares inadequados, onde essas têm consumido de forma excessiva produtos gordurosos, açúcares, doces e bebidas açucaradas e quantidade reduzida de frutas e verduras.

Essa mudança no padrão alimentar foi demonstrada pela POF (2008-2009), realizada pelo IBGE, que apontou aumento no consumo de carnes, frutas, bebidas e infusões e redução do consumo de cereais, leguminosas, oleaginosas, aves e ovos o que tem refletido nos altos índices de obesidade infantil e doenças associadas (IULIANO; MANCUSO; GAMBARDELLA, 2009; AIRES et al., 2011; SILVA; BITTAR, 2012).

\section{CONCLUSÃO}

Com o estudo foi possível conhecer a percepção dos pais de crianças entre 4 e 5 anos com relação ao nível de atividade física e hábitos alimentares da Escola Municipal Infantil Balão Mágico de Uruana de Minas.

Entende-se a importância de se abordar determinado assunto por se tratar de questões que englobam o desenvolvimento cognitivo da criança, alimentação e atividade física estando equilibrados garantem a elas condições favoráveis de progressão psicomotores associados a níveis mais saudáveis de vida, além disso, aos pais e escola entendimento da educação nutricional como ferramenta efetiva da saúde dentro e fora do contexto escolar.

A essa importância, uma vez que é na infância que os hábitos alimentares se formam, é necessário o entendimento dos seus fatores determinantes, para que seja 
possível propor intervenções educativas efetivas, para que ocorram mudanças nos padrões alimentares das crianças e consequentemente impacto positivo na qualidade de vida desta população.

\section{REFERÊNCIAS BIBLIOGRÁFICAS}

ANGELIS, R.C. Riscos e prevenção da obesidade: Fundamentos Fisiológicos e Nutricionais para tratamento. São Paulo: Atheneu, 2003.

AZEVEDO, Fernanda Reis de; BRITO, Bruna Cristina. Influência das variáveis nutricionais e da obesidade sobre a saúde e o metabolismo. Revista Associação Medicina Brasileira. v. 58. N 6. 2012. pp. 714-723.

ALVES, J.G.B. Sobrepeso em crianças atendidas em um consultório pediátrico privado do Recife. São Paulo: Pediatria, v. 6, 1984, p. 6973. Artigo http://queconceito.com.br/atividade-fisica

Abrantes MM, Lamounier JA, Colosimo EA. Prevalência de sobrepeso e obesidade em crianças e adolescentes das regiões Sudeste e Nordeste. J Pediatr (Rio J). 2002; 78:335-40.

BRASIL. Ministério do Desenvolvimento Social e Combate à Fome. Secretaria Nacional de Segurança Alimentar e Nutricional. Marco de Referência de Educação Alimentar e Nutricional para as Políticas Públicas. Brasília-DF: Ministério da Saúde, 2012.

BANKOFF, A.D.P. Obesidade e suas relações com as doenças crônicas degenerativas. 40 Congresso Londrinense de Ciências Biológicas Aplicadas à Saúde. Londrina, 2001.

BANKOFF, A.D.P.; ZAMAI, CA.; SCARPA, E.M; SCHMIDT, A; CIOL, P; FERRO, F; ROCHA, J. Estudo dos hábitos diários e antropometria de sujeitos com distúrbios de obesidade

BRASIL. Ministério do Desenvolvimento Social e Combate à Fome. Secretaria Nacional de Segurança Alimentar e Nutricional. Marco de Referência de Educação Alimentar e Nutricional para as Políticas Públicas. Brasília-DF: Ministério da Saúde, 2012. 
BARBOSA, V.L.P. Prevenção da obesidade na infância e na adolescência: exercício, nutrição e psicologia. São Paulo: Manole, 2004.

BEULKE, C. S. A Influência do Consumidor Infantil Sobre os Pais na Tomada de Decisão de Compra de Produtos Alimentícios. In: Intercom Júnior - Sociedade Brasileira de Estudos Interdisciplinares da Comunicação, XXVIII Congresso Brasileiro de Ciências da Comunicação - Uerj, 5 a 9 set., 2005. Disponível em: < http://www.portcom.intercom.org.br/pdfs/15347837636278613211572487682266596 2680. pdf>. Acesso em: 16 nov. 2014.

BARROS, Jussara de. Educação Física: Corpo em movimento disponível no sait: http://www.pedagogiaaopedaletra.com/posts/educacao-fisica-corpo-em movimento/acesso dia 10 setembro de 2011.

BERTOLETTI, Juliana; SANTOS, Seille Cristine Garcia. Avaliação do Estresse na Obesidade Infantil. Psico, Porto Alegre, PUCRS, v. 43, n. 1. 2012. pp. 32-38.

CAMPANA, Nathalia T.C; GOMES, Isabel C.; LERNER, Rogério. Contribuições da clínica da parentalidade no atendimento de um caso de obesidade. Revista pisc. Clin. v.26, n. 2. 2014. pp.105-119.

COSTA, Miguel Ataíde Pinto da; SOUZA, Marcos Aguiar de; OLIVEIRA, Valéria Marques de;Obesidade infantil e bullying: a ótica dos professores. Educação e

Pesquisa, São Paulo, v. 38, n. 03. 2012. pp. 653-665.

COELHO, Helena Martins; PIRES, António Prazo. Relações Familiares e Comportamento Alimentar. Psicologia: Teoria e Pesquisa. v30, n. 1.2014. pp. 45-52. COLLOCA, Edson Aparecido; DUARTE, Ana Claudia Garcia. OBESIDADE INFANTIL: Etiologia E Encaminhamentos, Uma Busca Na Literatura. Disponível em: <http://www.ufscar.br/ efe/pdf/2a/colloca.pdf> Acesso em: Novembro de 2015.

DeLany J. P.; Harsha, D. W.; Kime, J. C.; Kumler, J.; Melancon, L. \& Bray, G. a (1995). Energy expenditure in lean and obese prepubertal children. Obesity Research. 3, 67-72.

DeLany, J. P. (1998). Role of energy expenditure in thedevelopmentof pediatric obesity. American Journal of Clinical Nutrition. 68 (suppl), 950s-955s. 
Dietz, W. H. \& Gortmaker, S.L. (1985). Do we fatten our children at the television set? Obesity and television viewing in children and adolescents. Pediatrics, 75, 807812.

DIEZ-GARCIA, Rosa W.; CERVATO-MANCUSO Ana Maria.Mudanças alimentares e educação nutricional. Rio deJaneiro: Guanabara Koogan, 2012.

Dietz, W. H. (1994). Critical periods inchildhood for the development of obesity. American Journal Clinical Nutrition,59(5), 955-959.

Dietz, Willian H. (1995). Childhood obesity. In Lilian W. Y. Cheung \& Julius B. Richmond (ed.). Child, Health, Nutrition and Physical Activity. Champaign: Human Kinetics Publishers

DAMÂSCO, A. Obesidade. Rio de Janeiro: Editora Médica e Cientifica 2003. pp. 629.

DAMIANI, D; CARVALHO, DP; OLIVEIRA RG. Obesidade na infância: um grande desafio. Pediatr Mod, v. 36, 2000. p. 489-528.

DIETZ WH. Periods of risk in childhood for development ofadult obesity - What do we need to learn?J Nutr, v. 127, 1997,pp. 1884-6.

DIETZ, W.H. Therapeutic strategies in childhood obesity.Horm. Res.v.39, n.3, p.86, 1993.

DIETZ, W.H.: Preventing obesity in children and adolescents. Annu. Rev. Public. Health., v.22, n.3, p.37-53, 2001.

DRESCH, Franciele et al. Nível de atividade física de escolares da rede pública de ensino de um município do Rio Grande do Sul. Revista Brasileira em Promoção da Saúde, v. 26, n. 3, 2013.

GARCÍA, Magdalena Cuenca; et al. Clustering of Multiple Lifestyle Behaviors and Health-related Fitness in European Adolescents. Journal of Nutrition Education and Behavior, v. 45, n. 6, 2013.

GIORDANI, L.G.; ALMEIDA, C.S.; PACHECO, A.M.. Avaliação das oportunidades de desenvolvimento motor na habitação familiar de crianças entre 18 e 42 meses. Motri, Vila Real, v. 9, n. 3, jul.2013. 
JAIME, Patrícia Constante et al.Prevalência e distribuição sócio demográfica de marcadores de alimentação saudável, Pesquisa Nacional de Saúde, Brasil 2013.Epidemiol. Serv.,Brasília, v.24,n. 2.2015. pp. 267-276.

LOURENÇO, Margarida; SANTOS, Célia; CARMO, Isabel. Estado nutricional e hábitos alimentares em crianças de idade pré-escolar. Revista de Enfermagem. Coimbra. v. 4, n.1. 2014. pp. 7-14.

MOREIRA, T. Consumo alimentar fora de casa e sua adequação em crianças de idade pré-escolar. 2013. 79 f. Dissertação (Mestrado) -Faculdade de Ciências da Nutrição e Alimentação da Universidade do Porto, Portugal, 2013.

MORAES, P. M. DIAS, C. M. de S. B. Obesidade Infantil a Partir de um Olhar Histórico Sobre Alimentação. Interação Psicol, Curitiba, v. 16, n. 2, p. 317-326, jul./dez. 2012.

MACHADO, Juliana Costa; COTTA, Rosângela Minardi Mitre; SILVA, Luciana Saraiva da Abordagem do desvio positivo para a mudança de comportamento alimentar: revisão sistemática. Ver Panam Salud Publica, Washington, v.36,n. 2. 2014. pp. 134-140.

MAGALHÃES, Elma Izze da Silva et al. Perímetro da cintura, relação cintura/estatura e perímetro do pescoço como parâmetros na avaliação da obesidade central em crianças. Revista Paulista de Pediatria. v. 32, n. 3, 2014. pp.273-282.

MIZIARA, Ângela Maria Borges; VECTORE, Celia. Excesso de peso em escolares: percepções e intercorrências na escola. Revista Quadrimestral da Associação Brasileira de Psicologia Escolar e Educacional. São Paulo. V.18, N.2. 2014. pp. 283291.

MORAES, Leonardo Lezzi et al. Pressão Arterial Elevada em Crianças e sua Correlação com Três Definições de Obesidade Infantil. Arq Bras Cardiol. v.102, n. 2. 2014. pp. 175-180.

MORAES, Priscilla Machado; DIAS, Cristina Maria de Souza Brito. Obesidade Infantil a Partir de um Olhar Histórico Sobre Alimentação. Interação Psicol, Curitiba. v. 16, n. 2. 2012. pp. 317-326.Martins, S. (2005). Obesidade infantil, Medicina e Saúde, n.ำ 95, 9: 52-54. 
MORAES, Priscilla Machado; DIAS, Cristina Maria de Souza Brito.Nem Só de Pão se Vive: A Voz das Mães na Obesidade Infantil. Psicologia: ciência e profissão. V.33, n.1. 2013. pp.46-59.

MOREIRA, Mariana de Sousa Farias et.al. Doenças associadas à obesidade infantil. Revista Odontológica de Araçatuba, v.35, n.1. 2014. pp. 60-66. OMS - World Health Organization. Obesity. Disponível em: http://www.who.int/en/. Acesso em: 02/07/2012.

OLIVEIRA, Fabiana Azeredo de; MARTINS, Karla Patrícia Holanda. Implicações subjetivas da relação mãe criança nos quadros de obesidade infantil. Estilos da Clínica. 2012. V.7. N.1. pp. 122-135.

PIMENTA, T. A. M; ROCHA, R. MARCOndeS, N. A. V. Políticas Públicas de Intervenção na Obesidade Infantil no Brasil: uma Breve Análise da Política Nacional de Volume 16,Número 2ISSN 2447-2131João Pessoa, 2016 Obesidade infantil: influência dos pais sobre a alimentação e estilo de vida dos filhos Páginas 460a 481481 Alimentação e Nutrição e Política Nacional de Promoção da SaúdeUNOPAR Cient Ciênc Biol Saúde2015;17(2):139-46

PANDOLFO, Kelly Christine Maccarini et al. Estado Nutricional de Escolares do Ensino Público Federal. Revista Brasileira de Nutrição Esportiva, São Paulo. v. 8. n. 47. p. 300-306. Set./Out. 2014.

PRESCOTT, John. Chemosensory learning and favour: Perception, preference and intake. Physiology \& Behavior, v. 107, p. 553-559, 2012.

Rev. Bras. Ciênc. Esporte, Florianópolis, v. 35, n. 2, p. 441-453, abr./jun. 2013 441 Rev Paul Pediatr 2008; 26(3): 271-7http://www.scielo.br/pdf/rpp/v26n3/12

ROLLS, Edmund T. Taste, olfactory and food texture reward processing in the brain and the control of appetite.Proceedings oh the Nutrition Society -Department of Computer Science, Oxford Centre for Computational Neuroscience, Oxford, v. 71, p. 488-501, september 2012.

SANTOS, L. A. S. O fazer educação alimentar e nutricional: algumas contribuições para reflexão. Ciência \& Saúde Coletiva, v. 17, n. 2, p. 453-462, 2012.

SILVA, A. R. V et al. Hábitos alimentares de adolescentes de escolas públicas de Fortaleza, CE, Brasil. Rev. bras. enferm, v. 62, n. 1, p. 18-24, 2009. 
SENA, R.A; PRADO, S.R.L.A. Obesidade infantil relacionada a hábitos de vida e práticas alimentares. Rev EnfermUNISA. 2012; 13(1): 69-73.

Toni V, Gavineski IC, Migon P, Finato S, Rech RR, Halpen R. Insatisfação com a Imagem Corporal em Adolescentes de Escolas Públicas de Caxias do Sul - RS. Rev Bra Ciênc Saúde. 2012; 16:187-194.

VENÂNCIO, Paulo, Sara; PINTO, Graciete. Obesidade infantil... Um problema cada vez mais atual. Rev Port Med Geral Fam.v.28, N.4. 2012. pp.10-6. 


\section{ANEXOS}

\section{ANEXO I - SOBRE QUESTIONÁRIO APLICADO NA PESQUISA}

O questionário abaixo descrito apresenta perguntas elaboradas visando o contexto (realidade) estudado, foram necessárias algumas adaptações para que pudesse chegar ao resultado esperado.

\section{Questionário respondido por}

Pai ( ) Mãe ( ) Irmão ( )

Outro qual?

\section{Nome da Criança}

Sexo: Masculino ( ) Feminino ( )

1. A criança sofre de alguma patologia ou doença?

$\operatorname{Sim}($ ) Não ( )

2. Quantas horas por dia, a criança passa na escola? horas.

3. Da seguinte escala manifeste o seu nível de satisfação relativamente à criança na escola.

Pouco satisfeito (PS) Satisfeito(S) Muito Satisfeito (MS)
(A) Aulas de Educação Física que a criança tem na escola ( )
(B) Avalie o nível de atividade física oferecida pelo professor e a escola ( )
(C) Alimentação que é oferecida na escola ( )
(D) Serviços de apoio de nutrição/alimentação ( )

4. Como é que a criança se desloca para a escola? Assinale com uma cruz o(s) meio(s) em que se desloca e o tempo aproximado que demora em cada um.

\begin{tabular}{|l|l|l|l|l|}
\hline Nunca & 15 min & $\begin{array}{l}\text { Entre 15 a } \\
30 \mathrm{~min}\end{array}$ & $\begin{array}{l}\text { Mais que 30 } \\
\min \end{array}$ \\
\hline
\end{tabular}




\begin{tabular}{|l|l|l|l|l|}
\hline Á pé & & & & \\
\hline Bicicleta & & & & \\
\hline Carro & & & & \\
\hline Outros & & & & \\
\hline & & & & \\
\hline & & & & \\
\hline
\end{tabular}

5. Quanto tempo a criança, independente do sexo, se dedica em média (minutos ou horas), a ver TV, joga no celular, computador ou vídeo game ao longo da semana.

( ) min total na semana

( ) horas total na semana

6. Quantas vezes por mês, normalmente o seu filho/a come fora de casa. (não contar com as refeições da escola). Se nenhuma, coloque 00.
(A) Em casa de familiares ( ) ( ) vezes
(B) Em restaurante (fast-food, pizzarias e lanchonetes) $($ ) ( ) vezes

7. Durante o último ano teve dificuldade para consegui que a criança comesse o que desejava para ela saudável (considere frutas, verduras e legumes) no período em que não esta na escola.
(A) Sim, muita dificuldade
(B) Sim, alguma dificuldade
(C) Sim, ocasionalmente
(D) Não, nenhuma dificuldade ( ) 
